\title{
Individualized $\mathrm{HbA}_{1 \mathrm{c}}$ Goals, and Patient Awareness and Attainment of Goals in Type 2 Diabetes Mellitus: A Real-World Multinational Survey
}

\author{
Dominik Lautsch · Robert Boggs (D) - Tongtong Wang · \\ Claudio Gonzalez • Gary Milligan (D) - Swapnil Rajpathak • \\ Seema Malkani · Euan McLeod · James Carroll (D) · Victoria Higgins (iD
}

Received: September 30, 2021 / Accepted: November 2, 2021 / Published online: December 24, 2021

(c) The Author(s) 2021

\begin{abstract}
Aims: While glycemic control is key in effective type 2 diabetes mellitus management, many patients fail to reach their individualized glycemic goal. This analysis aimed to describe a real-world picture of diabetes management: individualized hemoglobin $\mathrm{A}_{1 \mathrm{c}}\left(\mathrm{HbA}_{1 \mathrm{c}}\right)$ goals, rate of goal attainment, $\mathrm{HbA}_{1 \mathrm{c}}$ at each line of therapy, and patient awareness of their glycemic goal. Secondly, we aimed to understand physician satisfaction with $\mathrm{HbA}_{1 \mathrm{c}}$ amongst patients aware vs. those unaware of $\mathrm{HbA}_{1 \mathrm{c}}$ goal. Methods: Analysis of physicians and the next ten consulting patients with type 2 diabetes mellitus conducted in Europe and the USA including medical record data abstraction/ assessment by physicians, a patient-reported survey and a physician survey. Patients were
\end{abstract}

Supplementary Information The online version contains supplementary material available at https:// doi.org/10.1007/s12325-021-01985-3.

D. Lautsch · R. Boggs · T. Wang · C. Gonzalez ·

S. Rajpathak $\cdot$ S. Malkani

Merck \& Co., Inc, Kenilworth, NJ, USA

G. Milligan · J. Carroll · V. Higgins ( $₫)$

Adelphi Real World, Adelphi Mill, Grimshaw Lane,

Bollington, Macclesfield SK10 5JB, Cheshire, UK

e-mail: Victoria.Higgins@adelphigroup.com

E. McLeod

Pfizer Ltd, Tadworth, UK diagnosed for 3 months or more with a known current and target $\mathrm{HbA}_{1 \mathrm{c}}$. For the sub-analysis assessment of patient awareness of $\mathrm{HbA}_{1 \mathrm{c}}$ goal, in addition to the above, these patients had to have completed a patient-reported questionnaire and answer the question on awareness of $\mathrm{HbA}_{1 \mathrm{c}}$ goal.

Results: A total of 730 physicians provided data on 8794 patients with type 2 diabetes mellitus; 5331 patients were eligible for this analysis. Overall, mean (standard deviation, SD) individualized $\mathrm{HbA}_{1 \mathrm{c}}$ goal was $6.8 \%(0.68 \%)$. Of eligible patients, $39.1 \%$ met their $\mathrm{HbA}_{1 \mathrm{c}}$ goal; of $60.9 \%$ of patients not reaching their $\mathrm{HbA}_{1 \mathrm{c}}$ goal, the mean distance from individualized $\mathrm{HbA}_{1 \mathrm{c}}$ goal was $0.9 \%$ (SD 1.0\%). Physicians progressed patients' antihyperglycemic therapy when $\mathrm{HbA}_{1 \mathrm{c}}$ was $8 \%$ or higher. Among 2560 patients who were included in the sub-analysis assessing the effect of patient awareness of their $\mathrm{HbA}_{1 \mathrm{c}}$ goal on multiple parameters, $70.5 \%$ were aware of their $\mathrm{HbA}_{1 \mathrm{c}}$ goal; mean $\mathrm{HbA}_{1 \mathrm{c}}$ goal was $6.8 \%$ $(0.7 \%)$ and current mean $\mathrm{HbA}_{1 \mathrm{c}}$ value $7.1 \%$ $(1.2 \%)$. A total of 949 patients in the sub-analysis (39.2\%) achieved their goal; achieving $\mathrm{HbA}_{1 \mathrm{c}}$ goal was not related to knowledge of goal. Patients aware of their $\mathrm{HbA}_{1 \mathrm{c}}$ goal were slightly more adherent to their antihyperglycemic medication. They also were prescribed more antihyperglycemic agents, more often on a later therapy line receiving a GLP-1 receptor agonist, SGLT2i, or insulin, and more often tested their blood glucose levels than patients 
who were unaware. Physicians were not satisfied with the current blood glucose level of one third of their patients, believing that more of those who were aware of their $\mathrm{HbA}_{1 \mathrm{c}}$ goal could achieve better glucose control (32.4\% of aware vs. $28.2 \%$ of unaware patients; $p=0.003$ ).

Conclusions: Our results showed that the proportion of patients with type 2 diabetes mellitus achieving their goals for glycemic control was suboptimal when compared to current guideline criteria, with only about $40 \%$ of patients achieving their individualized $\mathrm{HbA}_{1 \mathrm{c}}$ goal. Treatment intensification was often delayed until $\mathrm{HbA}_{1 \mathrm{c}}$ was $8 \%$ and higher. Patients aware of their $\mathrm{HbA}_{1 \mathrm{c}}$ goal were slightly more adherent to their antihyperglycemic medication; however, awareness of $\mathrm{HbA}_{1 \mathrm{c}}$ goal did not enhance goal attainment. This highlights the need for a holistic approach to diabetes management, involving patient education, and patient-physician communication and partnership.

Keywords: Glycemic control; Glycemic goal attainment; Individualized $\mathrm{HbA}_{1 \mathrm{c}}$ goal; Individualized $\mathrm{HbA}_{1 c} ;$ Individualized $\mathrm{HbA}_{1 \mathrm{c}}$ target; Patient goal awareness; Patient target awareness; Real world; Type 2 diabetes mellitus

\begin{tabular}{|c|c|}
\hline \multicolumn{2}{|c|}{ Abbreviations } \\
\hline AHA & Antihyperglycemic agent \\
\hline ARMS-D & Adherence to Refills and Medicines \\
\hline & Scale for Diabetes \\
\hline BMI & Body mass index \\
\hline CKD & Chronic kidney disease \\
\hline COVID-19 & Coronavirus disease \\
\hline CS & Chi-squared test \\
\hline DSP & Disease Specific Programme ${ }^{\mathrm{TM}}$ \\
\hline FE & Fisher's exact test \\
\hline GLP-1 & Glucagon-like peptide 1 \\
\hline $\mathrm{HbA}_{1 \mathrm{c}}$ & Hemoglobin $A_{1 c}$ \\
\hline PCP & Primary care physician \\
\hline SD & Standard deviation \\
\hline SGLT2i & $\begin{array}{l}\text { Sodium glucose cotransporter } 2 \\
\text { inhibitor }\end{array}$ \\
\hline SU & Sulfonylurea \\
\hline TT & $t$ test \\
\hline UK & United Kingdom \\
\hline USA & United States of America \\
\hline
\end{tabular}

\section{Key Summary Points}

What is already known about this subject?

Whilst guidelines recommend $\mathrm{HbA}_{1 \mathrm{c}}$ targets of $<7 \%$ for a majority of patients to reduce rates of development and progression of micro/macrovascular complications, individualized $\mathrm{HbA}_{1 \mathrm{c}}$ goals can vary in realworld clinical practice, with many patients failing to achieve their goal.

Evidence suggests that the proportion of patients maintaining target glycemic levels decreases over time, regardless of antihyperglycemic therapy.

Patient knowledge of glycemic goal is associated with better glycemic control.

\section{What is the key question?}

What is the real-world $\mathrm{HbA}_{1 \mathrm{c}}$ goal for patients with type 2 diabetes mellitus and how does patient awareness impact attainment?

\section{What are the new findings?}

Around $60 \%$ of patients were not at the $\mathrm{HbA}_{1 \mathrm{c}}$ goal set by their physicians, which was $6.8 \%$ on average; mean distance from $\mathrm{HbA}_{1 \mathrm{c}}$ goal was $0.9 \%$.

With each successive therapy line, physicians tended to wait until $\mathrm{HbA}_{1 \mathrm{c}}$ was progressively higher before changing/ adding pharmacologic agents.

Patients aware of their $\mathrm{HbA}_{1 \mathrm{c}}$ goal were slightly more adherent to their antihyperglycemic medication; however, awareness of $\mathrm{HbA}_{1 \mathrm{c}}$ goal did not enhance goal attainment.

\section{How might this impact on clinical practice in the foreseeable future?}

Results of this analysis highlighted the need for a holistic approach to diabetes management, involving patient education, and patient-physician communication and partnership. 


\section{INTRODUCTION}

Glycemic control is the primary goal of diabetes treatment to prevent target organ damage and other disease-related complications. Guidelines recommend a target glycated hemoglobin $\left(\mathrm{HbA}_{1 \mathrm{c}}\right)$ value of less than $7.0 \%$ $(<53 \mathrm{mmol} / \mathrm{mol})$ for most non-pregnant adults, although these targets are individualized per patient [1-3]. Target values need to be determined individually per patient by the treating physician, with factors influencing this decision including age, comorbidities and complications, or disease duration [3]. Lower $\mathrm{HbA}_{1 \mathrm{c}}$ levels have been observed to reduce rates of development and progression of microvascular complications $[4,5]$, to maintain reduction in microvascular risk [6], and reduce macrovascular complications [7, 8]. Guidelines also recommend that $\mathrm{HbA}_{1 \mathrm{c}}$ goals are individualized on the basis of patient characteristics, patient preferences and goals, and risk of treatment-related adverse effects such as hypoglycemia and weight gain [1, 2]. Previous realworld studies have focused on the prevalence of adults with type 2 diabetes mellitus achieving an $\mathrm{HbA}_{1 \mathrm{c}}$ goal of $<7.0 \%$ despite the recommended individualization of glycemic goals [9-14].

Although knowledge of glycemic goals was associated with better glycemic control [15-17], patient knowledge of their individualized glycemic goals is not well understood on a systematic level $[18,19]$. It was estimated that only about one quarter of patients with diabetes understand the meaning of $\mathrm{HbA}_{1 \mathrm{c}}$ or can recall their most recent value $[16,20]$, while some thought that their $\mathrm{HbA}_{1 \mathrm{c}}$ values above $8 \%$ indicated good glycemic control [21]. Understanding patient experiences is essential [22].

The achievement of and distance to the individualized $\mathrm{HbA}_{1 \mathrm{c}}$ goal, and the $\mathrm{HbA}_{1 \mathrm{c}}$ level at which providers intensify patients' treatment regimens by adding or changing to a second or third antihyperglycemic agent (AHA), have not been well characterized. The objectives of this analysis were to (1) describe individualized $\mathrm{HbA}_{1 \mathrm{c}}$ goal and rate of goal attainment; (2) note $\mathrm{HbA}_{1 \mathrm{c}}$ levels as patients progressed through lines of therapy; (3) understand patient awareness of goal, and association between awareness and goal attainment; and (4) understand differences in number of AHAs, lines of therapy, glucose testing, and physician satisfaction with $\mathrm{HbA}_{1 \mathrm{c}}$ amongst patients aware vs. those unaware of their $\mathrm{HbA}_{1 \mathrm{c}}$ goal.

\section{METHODS}

\section{Survey Design}

Data were drawn from the Adelphi Diabetes Disease Specific Programme ${ }^{\mathrm{TM}}$ (DSP), a large, real-world survey of physicians and their patients conducted in Europe and the USA. The DSP comprised physician surveys and medical record data abstraction by physicians, matched with patient-reported surveys. Data were collected in Germany, Italy, Spain, the UK, and the USA between October 2018 and March 2019. Full DSP methodology has been published and validated [23-25].

Upon providing consent to participate, primary care physicians (PCPs) or diabetologists/ endocrinologists involved with the management and treatment of patients with type 2 diabetes mellitus (monthly workload $\geq 25$ and $\geq 50$ patients with type 2 diabetes mellitus, respectively) enrolled the next ten consecutive patients who presented in their offices and met the patient eligibility criteria, at least 18 years old, not in a clinical trial at time of data capture, and currently receiving at least one AHA. Physicians were also asked to include two additional patients treated with either a sodium glucose cotransporter 2 inhibitor (SGLT2i) or a glucagon-like peptide 1 (GLP-1) receptor agonist, or both of these agents (either alone or in combination with other AHAs), to ensure newer AHAs were represented. The research methodology is designed to maximize the number of physicians sampled while minimizing the burden on each physician by limiting the number of patients on whom they report. This also increases the power of the sample size overall. For each patient who met the eligibility criteria, physicians completed a form containing detailed questions, capturing patient 
demographics, tests performed (including current $\mathrm{HbA}_{1 \mathrm{c}}$ value, i.e., "at time of data collection"), $\mathrm{HbA}_{1 \mathrm{c}}$ goal, comorbid conditions, and current and previous treatment including $\mathrm{HbA}_{1 \mathrm{c}}$ at time of initiation.

Physicians then invited the same patients to complete, on a voluntary basis, a patient-reported form, containing questions about demographics and current condition. Patients also answered the question "Do you have an agreed blood sugar target with your doctor?". In addition, information on medication adherence was reported using the Adherence to Refills and Medicines Scale for Diabetes (ARMS-D) [26, 27]. The ARMS-D is an 11-item self-report measure of adherence that assesses patients' ability to take and refill diabetes medications, generating total, refill, and medication-taking subscale scores. Each of the items is structured for a response on a 4-point Likert scale and scored as $1=$ "none," 2 = "some," 3 = "most," or 4 = "all" of the time, with higher values indicating poorer adherence (total score ranges from 11 to 44) ) $[26,27]$.

Physians also completed a workload form to record a 5-day period of overall patient caseload, including consultation of patients with T2DM, irrespective of patients recruited into the survey.

To be included in this retrospective analysis of the Diabetes DSP ${ }^{\mathrm{TM}}$, patients had to have a physician-reported current and target $\mathrm{HbA}_{1 \mathrm{c}}$, and have been diagnosed with type 2 diabetes mellitus for at least 3 months. For assessment of patient awareness of $\mathrm{HbA}_{1 \mathrm{c}}$ goal (the sub-analysis), in addition to the above, patients had to have completed a patient-reported questionnaire and answer the question on awareness of $\mathrm{HbA}_{1 \mathrm{c}}$ goal.

The survey obtained ethics approval from the Western Institutional Review Board, study protocol number 1247198, and was performed in accordance with the Helsinki Declaration of 1964 and its later amendments. Physicians did not see patient responses, thereby ensuring that future interactions between physicians and their patients were not compromised by patient responses. Patients provided written informed consent for use of their anonymized and aggregated data.

\section{Statistical Analysis}

Patient characteristics were summarized using descriptive analyses. Means and standard deviations (SDs) were calculated for continuous variables, and frequency and percentages were calculated for categorical variables. Glycemic control rates were calculated as the proportion of patients with a current $\mathrm{HbA}_{1 \mathrm{c}}$ level lower than the individualized $\mathrm{HbA}_{1 \mathrm{c}}$ goal set by physicians.

Inferential analyses were used to explore differences in patients' characteristics between those achieving and those not achieving individualized $\mathrm{HbA}_{1 \mathrm{c}}$ goals. Fisher's exact test was used for binary categorical variables, chisquared test was used for unordered categorical variables (more than two groups), and a $t$ test was used for continuous variables.

Multivariate regression was performed to determine the relationship between patient knowledge of their $\mathrm{HbA}_{1 \mathrm{c}}$ goal and goal achievement, controlling for age, gender, time since diagnosis, body mass index (BMI), previous $\mathrm{HbA}_{1 \mathrm{c}}$ value, Charlson Comorbidity Index (performed excluding diabetes as a comorbidity) [28], and number of AHAs currently used. A $p$ value of less than 0.05 was taken as statistically significant.

\section{RESULTS}

\section{Participant Characteristics}

\section{Physician Demographics}

A total of 730 physicians, 306 (58\%) PCPs and 424 (42\%) diabetologists/endocrinologists, provided data on 8794 patients with type 2 diabetes mellitus (Fig. 1). Country-level differences are reported in Supplementary Table 1.

\section{Patient Demographics}

Of 5331 patients eligible for the analysis, 1126 (54.0\%) patients were male with no differences across countries or previous waves of the survey. The median (IQR) duration of diabetes was 4.7 (2.0-9.6) years (Table 1). Physicians reported that after diabetes, the most common 


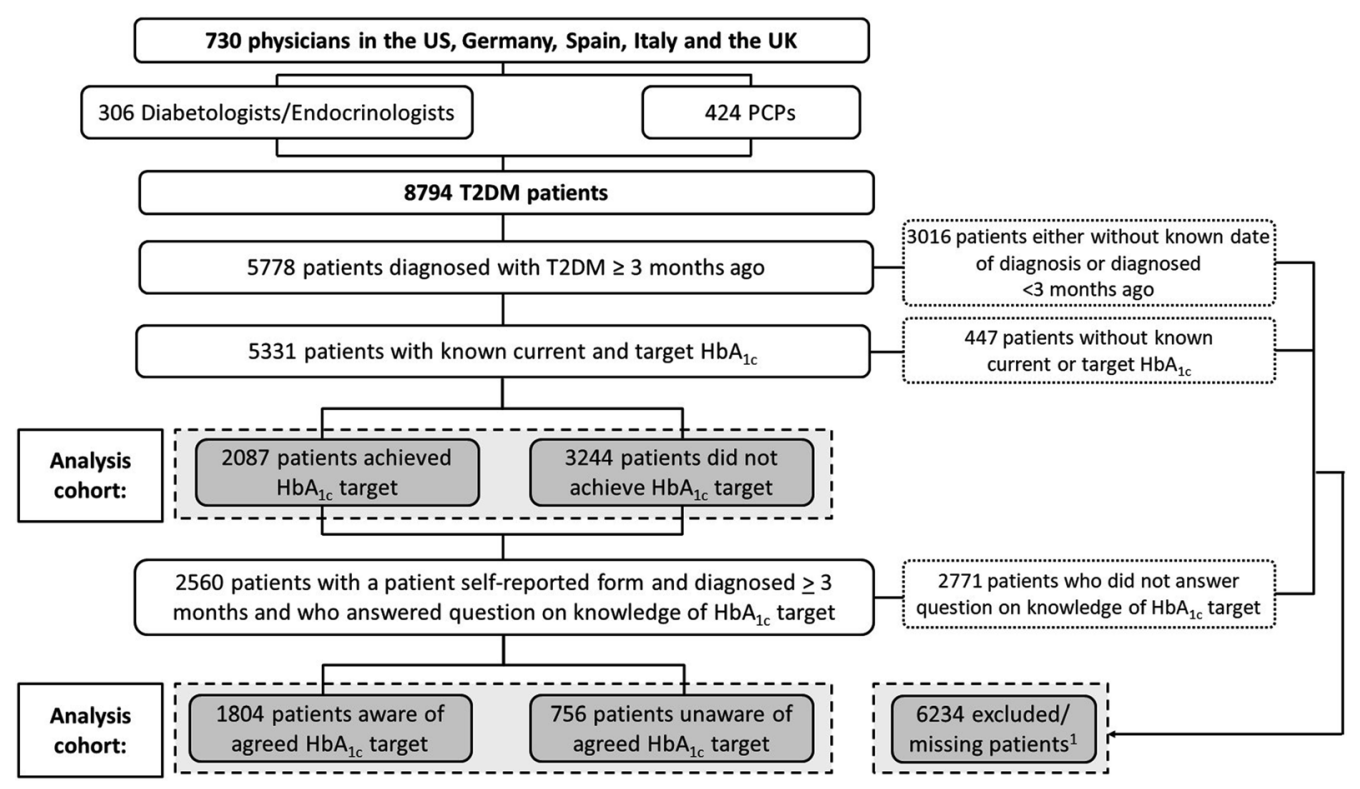

Fig. 1 Participant flow diagram. HbA $1 \mathrm{c}$ glycated hemoglobin, PCPs primary care physicians, T2DM type 2 diabetes mellitus. ${ }^{1}$ These patients did not have a known physician-reported current or target $\mathrm{HbA}_{1 \mathrm{c}}$, were diagnosed with type 2 diabetes mellitus less than 3 months ago, chose not to complete a self-reported record form, or chose

cardiovascular-metabolic comorbid condition across the patient cohort was hypertension (67.1\%), followed by CKD (6.8\%) and coronary artery disease $(6.6 \%)$.

Non-eligible patients are described in the Supplementary File.

\section{Individualized $\mathrm{HbA}_{1 \mathrm{c}}$ Goals, Patient Attainment of Their $\mathrm{HbA}_{1 \mathrm{c}}$ Goal and $\mathrm{HbA}_{1 \mathrm{c}}$ at Pharmacologic Change/ Addition}

\section{Individualized $\mathrm{HbA}_{1 c}$ Assessment}

Overall, patients had a mean (SD) individualized $\mathrm{HbA}_{1 \mathrm{c}}$ goal of $6.8 \%(0.68 \%)$. Of those patients with a known current and target $\mathrm{HbA}_{1 \mathrm{c}}$ $(n=5331), 39.1 \%$ met their individualized $\mathrm{HbA}_{1 \mathrm{c}}$ goal $(n=2087)$. Among those patients not reaching their individualized $\mathrm{HbA}_{1 \mathrm{c}}$ goal $(n=3244 ; 60.9 \%)$, the mean (SD, median) distance from individualized $\mathrm{HbA}_{1 \mathrm{c}}$ goal was $0.9 \%$ $(1.0 \% ; 0.6 \%)$. Individual country data also showed that patients in the UK had the longest disease duration and highest current $\mathrm{HbA}_{1 \mathrm{c}}$, not to answer the question on awareness of their $\mathrm{HbA}_{1 \mathrm{c}}$ goal. Patients may or may not have been aware of their $\mathrm{HbA}_{1 \mathrm{c}}$ goal

$\mathrm{HbA}_{1 \mathrm{c}}$ goal, and $\mathrm{HbA}_{1 \mathrm{c}}$ at pharmacologic change/addition compared with all other countries; Germany had the shortest disease duration and lowest $\mathrm{HbA}_{1 \mathrm{c}}$ at change/addition second lowest current $\mathrm{HbA}_{1 \mathrm{c}}, \mathrm{HbA}_{1 \mathrm{c}}$ goal, indicating good correlation between disease duration and the $\mathrm{HbA}_{1 \mathrm{c}}$ difference. Overall, characteristics of patients not achieving their $\mathrm{HbA}_{1 \mathrm{c}}$ goal were similar to those achieving their goal (Table 1).

\section{$\mathrm{HbA}_{1 c}$ at Change/Addition of Pharmacologic Agents by Line of Therapy}

Physicians' decision to change/add patients' pharmacologic agents at lines of therapy typically occurred above an $\mathrm{HbA}_{1 \mathrm{c}}$ of $8 \%$. Pharmacologic change/addition from first-line of therapy to second-, third-, and fourth-line of therapy was made when $\mathrm{HbA}_{1 \mathrm{c}}$ levels were $8.3 \%$ $(n=1998), 8.3 \%(n=535), 8.5 \%(n=116)$, and $9.1 \%(n=16)$, respectively (Fig. 2$)$. 
Table 1 Patient demographic and clinical characteristics by achievement of $\mathrm{HbA}_{1 \mathrm{c}}$ goal

\begin{tabular}{|c|c|c|c|c|}
\hline & $\begin{array}{l}\text { Overall } \\
(N=5331)\end{array}$ & $\begin{array}{l}\text { Patients achieving } \mathrm{HbA}_{1 \mathrm{c}} \\
\text { goal }(N=2087)\end{array}$ & $\begin{array}{l}\text { Patients not achieving } \mathrm{HbA}_{1 \mathrm{c}} \\
\text { goal }(N=3244)\end{array}$ & $p$ value \\
\hline \multicolumn{5}{|l|}{ Characteristic } \\
\hline Age, years, mean (SD) & $59.9(12.1)$ & $60.7(12.1)$ & $58.0(12.0)$ & $\begin{array}{c}<0.0001 \\
\quad(\mathrm{TT})\end{array}$ \\
\hline Male, $n(\%)$ & $2934(55.0)$ & $1126(54.0)$ & $1808(55.7)$ & $\begin{array}{r}0.2045 \\
(\mathrm{FE})\end{array}$ \\
\hline $\begin{array}{l}\text { Body mass index, } \mathrm{kg} / \mathrm{m}^{2} \text {, } \\
\text { mean }(\mathrm{SD})\end{array}$ & $30.1(5.5)$ & $29.7(5.3)$ & $30.4(5.6)$ & $\begin{aligned}< & 0.0001 \\
& (\mathrm{TT})\end{aligned}$ \\
\hline $\begin{array}{l}\text { Duration of diabetes, years, } \\
\text { median (IQR) }\end{array}$ & $4.7(2.0,9.6)$ & $4.3(2.0,8.9)$ & $4.9(2.0,10.0)$ & $\begin{array}{l}0.005 \\
\quad(\mathrm{MW})\end{array}$ \\
\hline \multicolumn{5}{|l|}{$\mathrm{HbA}_{1 \mathrm{c}}$ assessment, mean (SD) } \\
\hline $\begin{array}{l}\text { Individualized } \mathrm{HbA}_{1 \mathrm{c}} \\
\text { goal }\end{array}$ & $6.8(0.7)$ & $6.8(0.6)$ & $6.8(0.8)$ & $\begin{array}{r}0.1039 \\
(\mathrm{TT})\end{array}$ \\
\hline Most recent $\mathrm{HbA}_{1 \mathrm{c}}$ & $7.3(1.2)$ & $6.5(0.6)$ & $7.7(1.3)$ & $\begin{array}{c}<0.0001 \\
\quad(\mathrm{TT})\end{array}$ \\
\hline $\begin{array}{l}\text { Distance from } \\
\text { individualized } \mathrm{HbA}_{1 \mathrm{c}} \\
\text { goal }^{\mathrm{a}}\end{array}$ & $0.5(1.0)$ & $-0.3(0.3)$ & $0.9(1.0)$ & $\begin{array}{c}<0.0001 \\
\quad(\mathrm{TT})\end{array}$ \\
\hline \multicolumn{5}{|c|}{ Comorbidities (physician-reported), $n$ (\%) } \\
\hline Missing, $n$ & 1 & 0 & 1 & - \\
\hline Atrial fibrillation & $275(5.2)$ & $104(5.0)$ & $171(5.3)$ & $\begin{array}{r}0.6575 \\
(\mathrm{FE})\end{array}$ \\
\hline Chronic kidney disease & $362(6.8)$ & $147(7.0)$ & $215(6.6)$ & $\begin{array}{r}0.0811 \\
(\mathrm{FE})\end{array}$ \\
\hline $\begin{array}{l}\text { Coronary heart/arterial } \\
\text { disease }\end{array}$ & $351(6.6)$ & $129(6.2)$ & $222(6.8)$ & $\begin{array}{r}0.3654 \\
(\mathrm{FE})\end{array}$ \\
\hline Heart failure & $134(2.5)$ & $53(2.5)$ & $81(2.5)$ & $\begin{array}{r}0.9288 \\
(\mathrm{FE})\end{array}$ \\
\hline Hypertension & $3286(61.7)$ & $1348(64.6)$ & $1938(59.8)$ & $\begin{array}{r}0.0004 \\
(\mathrm{FE})\end{array}$ \\
\hline Post-myocardial infarction & $177(3.3)$ & $61(2.9)$ & $116(3.6)$ & $\begin{array}{r}0.2104 \\
(\mathrm{FE})\end{array}$ \\
\hline Peripheral vascular disease & $154(2.9)$ & $55(2.6)$ & $99(3.1)$ & $\begin{array}{r}0.4028 \\
(\mathrm{FE})\end{array}$ \\
\hline
\end{tabular}


Table 1 continued

\begin{tabular}{lcccr}
\hline & $\begin{array}{l}\text { Overall } \\
(\boldsymbol{N}=\mathbf{5 3 3 1})\end{array}$ & $\begin{array}{l}\text { Patients achieving } \mathbf{H b A}_{\mathbf{1 c}} \\
\text { goal }(\boldsymbol{N}=\mathbf{2 0 8 7})\end{array}$ & $\begin{array}{l}\text { Patients not achieving } \mathbf{H b A}_{\mathbf{1 c}} \\
\text { goal }(\boldsymbol{N}=\mathbf{3 2 4 4})\end{array}$ & $\boldsymbol{p}$ value \\
\hline Post-stroke & $46(0.9)$ & $25(1.2)$ & $21(0.6)$ & 0.0473 \\
& & & $(\mathrm{FE})$ \\
\hline
\end{tabular}

Total sample included where there are no missing data reported

$F E$ Fisher's exact test, $H b A_{1 c}$ glycated hemoglobin, $I Q R$ interquartile range, $M W$ Mann-Whitney, $S D$ standard deviation, $T T$ t test

${ }^{\mathrm{a}}$ For those patients who did not attain their $\mathrm{HbA}_{1 \mathrm{c}}$ goal

\section{Patient Awareness of Their Individual Glycemic Goal and Effect on Goal Attainment and Physician-Reported Assessment of Patient $\mathrm{HbA}_{1 \mathrm{c}}$ Testing}

Patient Demographics, $\mathrm{Hb}_{1 c}$ Value and Goal Of 5331 patients with type 2 diabetes mellitus diagnosed for at least 3 months with a known current and target $\mathrm{HbA}_{1 \mathrm{c}}, 2560$ (48.0\%) patients qualified for the sub-analysis of whether they were aware of their $\mathrm{HbA}_{1 \mathrm{c}}$ goal agreed with their physician.

Of these 2560 patients, 1804 (70.5\%) patients were aware of their $\mathrm{HbA}_{1 \mathrm{c}}$ goal. Overall, patients in this sub-analysis had a mean (SD) age of 58.4 (11.7) years and 1396 (54.5\%) were male (Table 2 ). There were no statistically significant differences in the demographics of patients who were aware and unaware of their $\mathrm{HbA}_{1 \mathrm{c}}$ goal. Overall, patients in the sub-analysis were currently using a mean of two AHAs, including metformin (62.5\%) and very longacting insulin (13.7\%).

As $70.9 \%$ of the patient sample $(6234 / 8794)$ did not qualify for this sub-analysis, demographics and outcomes were also compared between those qualifying and not qualifying for the sub-analysis (i.e., patients with an unknown

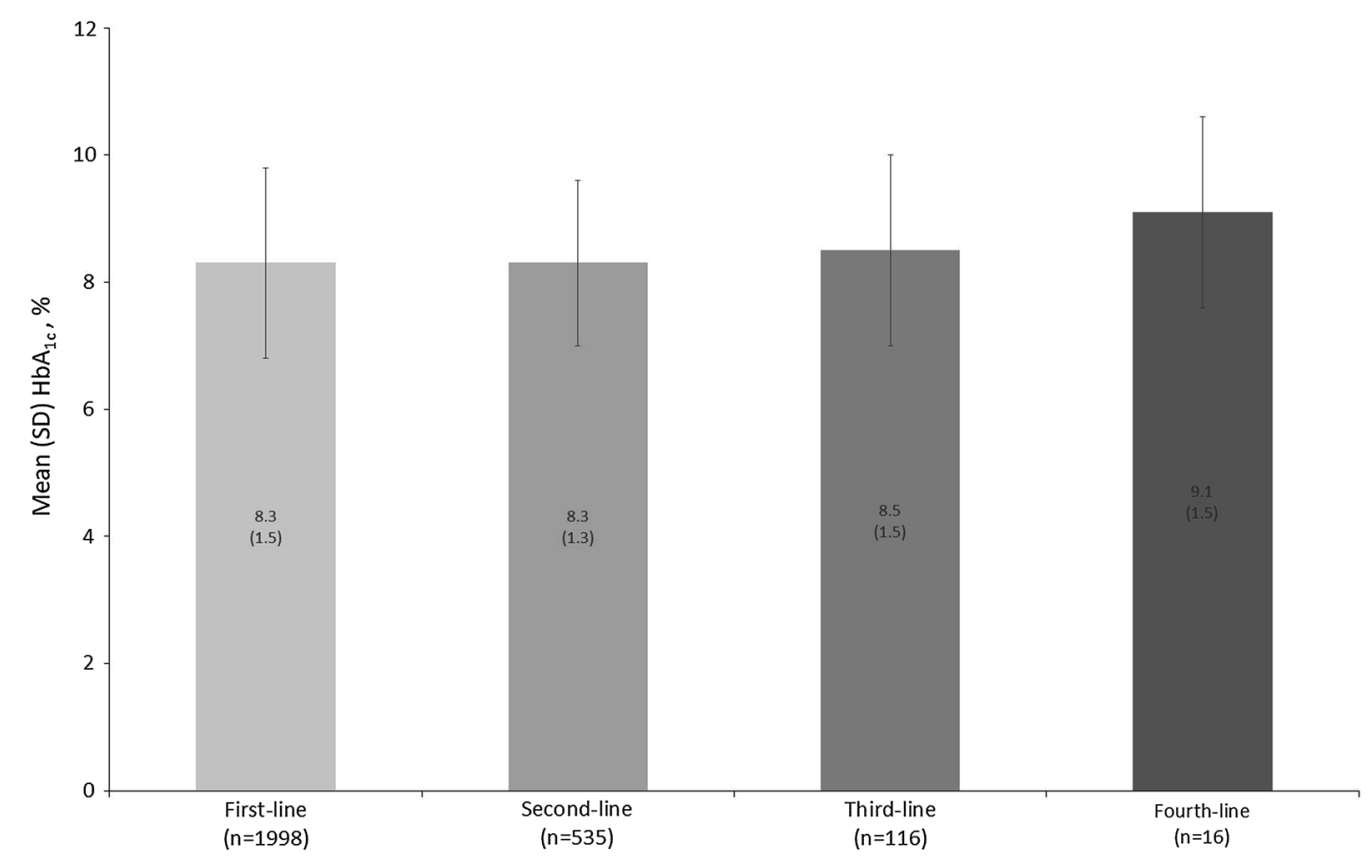

Fig. 2 Mean $\mathrm{HbA}_{1 \mathrm{c}}$ at change/addition of line of therapy. $\mathrm{HbA}_{1 \mathrm{c}}$ glycated hemoglobin, $\mathrm{SD}$ standard deviation. $\mathrm{HbA}_{\mathrm{lc}}$ value is physician-reported 
Table 2 Physician and patient characteristics by awareness of agreed $\mathrm{HbA}_{1 \mathrm{c}}$ goal

$\begin{array}{llll}\begin{array}{l}\text { Overall } \\ (N=2560)\end{array} & \begin{array}{l}\text { Patients unaware of agreed } \\ \mathrm{HbA}_{1 \mathrm{c}} \text { goal }(N=756)\end{array} & \begin{array}{l}\text { Patients aware of } \\ \mathrm{HbA}_{1 \mathrm{c}} \text { goal } \\ (N=1804)\end{array} & p \text { value }\end{array}$

Physician-level information

Physician characteristics

Physician specification $<0.0001$

Primary care physician

$1556(60.8) \quad 507(67.1)$

$1049(58.1)$

Specialist (diabetologist/

$1004(39.2) \quad 249(32.9)$

755 (41.9)

endocrinologist)

Physician qualifying year

$(\mathrm{CH})$

Before 1982

$147(5.7)$

$49(6.5)$

$98(5.4)$

1982-1994

$912(35.6) \quad 287(38.0)$

$625(34.6)$

1995-2004

$991(38.7) \quad 237(31.3)$

$754(41.8)$

2005-2015

$464(18.1) \quad 166(22.0)$

298 (16.5)

After 2015

46 (1.8)

17 (2.2)

$29(1.6)$

Physician 5-day workload, mean

$44.8(35.1)$

$43.2(35.4)$

$45.4(35.0)$

0.1341

patients with T2DM, $n$ (SD)

\section{Patient-level information}

\section{Patient demographics}

Age, years, mean (SD)

$$
\begin{array}{ll}
58.4(11.7) & 59.0(12.5) \\
1396(54.5) & 413(54.6)
\end{array}
$$

$58.2(11.3)$

0.0819

Male, $n(\%)$

$983(54.5)$

0.9653

Body mass index, $\mathrm{kg} / \mathrm{m}^{2}$, mean

$29.8(5.3)$

$29.9(5.3)$

$29.8(5.3)$

0.8081

(SD)

\section{Physician-reported clinical characteristics}

Median time since diagnosis, $\quad 4.3(1.9,8.7) \quad 4.4(2.0,8.9)$
years (IQR)

$4.3(1.9,8.6)$

0.4012

years (IQR)

(MW)

Mean most recent $\mathrm{HbA}_{1 \mathrm{c}}$

$\begin{array}{lll}\text { Missing, } n & 107 & 34 \\ \text { Mean \% (SD) } & 7.1(1.2) & 7.1(1.2)\end{array}$

73

$7.1(1.1)$

0.5659

Mean target $\mathrm{HbA}_{1 \mathrm{c}}$

Missing, $n$

31

18

13 
Table 2 continued

\begin{tabular}{|c|c|c|c|c|}
\hline & $\begin{array}{l}\text { Overall } \\
(N=2560)\end{array}$ & $\begin{array}{l}\text { Patients unaware of agreed } \\
\text { HbA }_{1 \mathrm{c}} \text { goal }(N=756)\end{array}$ & $\begin{array}{l}\text { Patients aware of } \\
\mathrm{HbA}_{\mathbf{1 c}} \text { goal } \\
(\boldsymbol{N}=\mathbf{1 8 0 4})\end{array}$ & $p$ value \\
\hline Mean \% (SD) & $6.8(0.7)$ & $6.8(0.9)$ & $6.8(0.7)$ & $\begin{array}{r}0.1705 \\
(\mathrm{TT})\end{array}$ \\
\hline \multicolumn{5}{|c|}{ At (or below) current $\mathrm{HbA}_{1 \mathrm{c}} \%$ goal } \\
\hline Missing, $n$ & 136 & 51 & 85 & \\
\hline$n(\%)$ & $949(39.2)$ & $292(41.4)$ & $657(38.2)$ & $\begin{array}{r}0.1430 \\
(\mathrm{FE})\end{array}$ \\
\hline \multicolumn{5}{|l|}{ Current therapy class, $n$ (\%) } \\
\hline Missing, $n$ & 1 & 1 & 0 & \\
\hline Metformin & $1600(62.5)$ & $499(66.1)$ & $1101(61.0)$ & $\begin{array}{r}0.0176 \\
(\mathrm{FE})\end{array}$ \\
\hline Metformin monotherapy & $669(26.1)$ & $267(35.4)$ & $402(22.3)$ & $\begin{aligned}< & 0.0001 \\
& (\mathrm{FE})\end{aligned}$ \\
\hline SU & $263(10.3)$ & $76(10.1)$ & $187(10.4)$ & $\begin{array}{r}0.8865 \\
(\mathrm{FE})\end{array}$ \\
\hline DPP4i & $259(10.1)$ & $80(10.6)$ & $179(9.9)$ & $\begin{array}{r}0.6152 \\
(\mathrm{FE})\end{array}$ \\
\hline SGLT2i & $407(15.9)$ & $101(13.4)$ & $306(17.0)$ & $\begin{array}{r}0.0243 \\
(\mathrm{FE})\end{array}$ \\
\hline GLP-1 & $510(19.9)$ & $98(13.0)$ & $412(22.8)$ & $\begin{aligned}< & 0.0001 \\
& (\mathrm{FE})\end{aligned}$ \\
\hline Very rapid-acting insulin & $134(5.2)$ & $32(4.2)$ & $102(5.7)$ & $\begin{array}{r}0.1727 \\
(\mathrm{FE})\end{array}$ \\
\hline Very long-acting insulin & $350(13.7)$ & $76(10.1)$ & $274(15.2)$ & $\begin{array}{r}0.0005 \\
(\mathrm{FE})\end{array}$ \\
\hline Any insulin & $532(20.8)$ & $114(15.1)$ & $418(23.2)$ & $<0.0001$ \\
\hline
\end{tabular}

Mean number of drugs in current regimen

$\begin{array}{llll}\text { Missing, } n & 1 & 1 & 0 \\ n(\mathrm{SD}) & 1.93(0.89) & 1.77(0.88) & 1.99(0.88)\end{array}$

Mean current line of therapy

$\begin{array}{lll}\text { Missing, } n & 467 & 115 \\ n(\mathrm{SD}) & 1.68(0.80) & 1.57(0.72)\end{array}$

352

$1.73(0.83)<0.0001$ 
Table 2 continued

\begin{tabular}{lllll}
\hline & $\begin{array}{l}\text { Overall } \\
(\boldsymbol{N}=\mathbf{2 5 6 0})\end{array}$ & $\begin{array}{l}\text { Patients unaware of agreed } \\
\mathbf{H b A}_{\mathbf{1 c}} \text { goal }(\boldsymbol{N}=\mathbf{7 5 6})\end{array}$ & $\begin{array}{l}\text { Patients aware of } \\
\mathbf{H b A}_{\mathbf{1 c}} \text { goal } \\
(\boldsymbol{N}=\mathbf{1 8 0 4})\end{array}$ & $\boldsymbol{p}$ value \\
\hline $\begin{array}{l}\text { Charlson Comorbidity Index } \\
\text { score (SD) }\end{array}$ & $0.3(0.9)$ & $0.4(0.9)$ & $0.3(0.8)$ & 0.1758 \\
$(\mathrm{TT})$
\end{tabular}

Patient-reported adherence and therapy satisfaction

Mean total ARMS-D score (range 11-44) ${ }^{\text {b }}$

$\begin{array}{lllll}\text { Missing, } n & 73 & 19 & 54 & \\ \text { Mean (SD) } & 15.0(3.7) & 15.5(3.9) & 14.8(3.6) & <0.0001 \\ \text { (TT) }\end{array}$

Mean ARMS-D refill subscale score (range 4-16)

$\begin{array}{lllll}\text { Missing, } n & 47 & 13 & 34 & \\ \text { Mean (SD) } & 6.2(1.7) & 6.5(1.7) & 6.1(1.7) & <0.0001 \\ & & & & (\mathrm{TT})\end{array}$

Mean ARMS-D medication-taking subscale score (range 7-28)

$\begin{array}{lllll}\text { Missing, } n & 58 & 15 & 43 & \\ \text { Mean (SD) } & 8.8(2.6) & 9.0(2.7) & 8.7(2.5) & 0.0108\end{array}$

0.0108
(TT)

\section{Physician-reported glucose testing}

Proportion of patients self-testing glucose levels

Missing, $n$
$n(\%)$ $1745(68.4) \quad 412(54.6)$ $1333(74.1) \quad<0.0001$

Are physicians satisfied with the patient's current blood glucose level? $n(\%)$ 0.0012

$(\mathrm{CH})$

\begin{tabular}{llll} 
Yes & $1494(58.4)$ & $455(60.2)$ & $1039(57.6)$ \\
$\begin{array}{l}\text { No, but this is the best that can } \\
\text { be realistically achieved }\end{array}$ & $236(9.2)$ & $88(11.6)$ & $148(8.2)$ \\
$\begin{array}{l}\text { No, and I believe that better } \\
\text { control can be achieved }\end{array}$ & $830(32.4)$ & $213(28.2)$ & $617(34.2)$ \\
\hline
\end{tabular}


Table 2 continued

\begin{tabular}{lll}
$\begin{array}{l}\text { Overall } \\
(\boldsymbol{N}=\mathbf{2 5 6 0})\end{array}$ & $\begin{array}{l}\text { Patients unaware of agreed } \\
\mathbf{H b A}_{\mathbf{1 c}} \text { goal }(\boldsymbol{N}=\mathbf{7 5 6})\end{array}$ & $\begin{array}{l}\text { Patients aware of } \\
\mathbf{H b A}_{\mathbf{1 c}} \text { goal } \\
(\boldsymbol{N}=\mathbf{1 8 0 4})\end{array}$ \\
\hline $\begin{array}{c}\text { Overall } \\
(N=2560)\end{array}$ & $\begin{array}{c}\text { Primary care physician } \\
(N=1556)\end{array}$ & Specialist $(N=1004)$ \\
\end{tabular}

$p$ value

Overall

Consulting physicin specialty

Consulting physician specialty

Patient-reported awareness of agreed $\mathrm{HbA}_{1 \mathrm{c}}$ goal, $n$ (\%)
Aware
$1804(70.5)$
$1049(67.4)$
$755(75.2)$
$<0.0001$

(FE)

ARMS-D Adherence to Refills and Medications Scale for Diabetes, $C S$ chi-squared test, DPP4i dipeptidyl peptidase 4 inhibitor, FE Fisher's exact test, GLP-1 glucagon-like peptide 1 receptor agonist, $H b A_{1 c}$ glycated hemoglobin, IQR interquartile range, $M W$ Mann-Whitney, $S D$ standard deviation, $S G L T 2 i$ sodium glucose cotransporter 2 inhibitors, $S U$ sulfonylurea, $T T$ t test

Total sample included where there are no missing data reported

${ }^{a}$ Scale of $0,1-2,3-4$, and $\geq 5$; each increasing level represents an increase in the cumulative mortality attributable to comorbid disease

${ }^{\mathrm{b}}$ Scale of 1 , none of the time to 4, and all of the time; higher ARMS total and subscale scores indicate poorer adherence

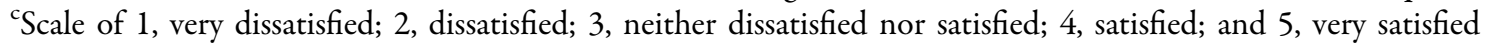

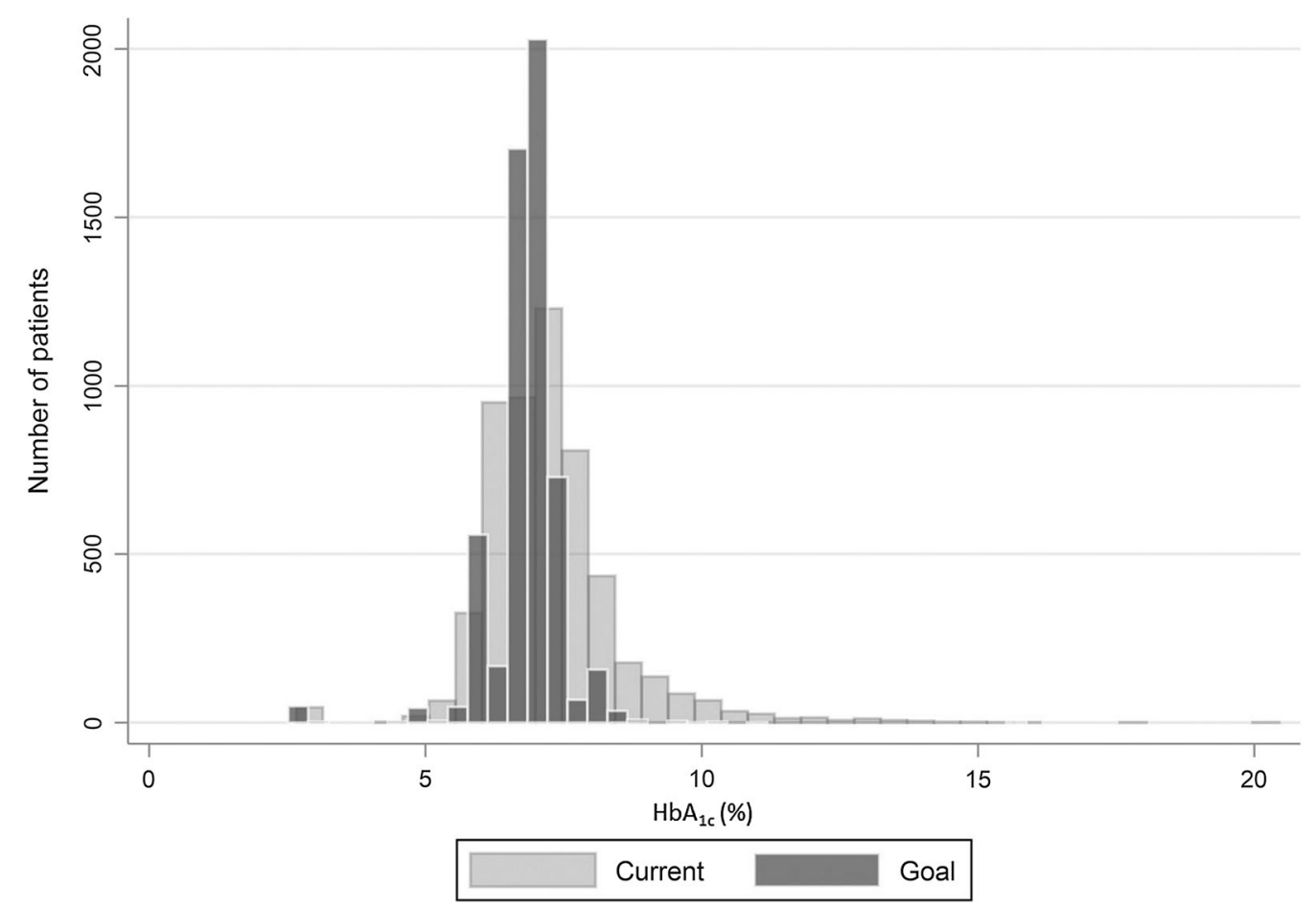

Fig. 3 Distribution of current and target $\mathrm{HbA}_{1 \mathrm{c}}$ among patients. $\mathrm{HbA}_{\mathrm{lc}}$ glycated hemoglobin 
$\mathrm{HbA}_{1 \mathrm{c}}$ goal, diagnosed with type 2 diabetes mellitus for less than 3 months, patients who did not chose to complete a self-reported record form, or those who chose not to answer the question on awareness of their $\mathrm{HbA}_{1 \mathrm{c}}$ goal) to investigate potential for bias. Minimal differences were seen between patients qualifying and not qualifying for this sub-analysis (Supplementary Table 2).

The individualized mean $\mathrm{HbA}_{1 \mathrm{c}}$ goal was $6.8 \%(0.7 \%)$ for the combined 2560 patients who were aware and unaware of their $\mathrm{HbA}_{1 \mathrm{c}}$ goal (Table 2). A higher proportion of patients aware of their $\mathrm{HbA}_{1 \mathrm{c}}$ goal were in the $6 \%$ to $7.5 \%$ patient groupings when compared to other HbA1c goal groupings i.e. $<6 \%$ and $7.5 \%+$ (Supplementary Table 3 ). Their current mean $\mathrm{HbA}_{1 \mathrm{c}}$ value was $7.1 \%$ (1.2\%). Distribution of current and target $\mathrm{HbA}_{1 \mathrm{c}}$ among patients is shown in Fig. 3.

\section{Awareness of Glycemic Goal and Effect on Goal Attainment}

Although the majority of patients included in the sub-analysis were aware of their $\mathrm{HbA}_{1 \mathrm{c}}$ goal, physicians reported that only 949 patients $(39.2 \%)$ of these patients were successful in achieving their goal.

Achieving $\mathrm{HbA}_{1 \mathrm{c}}$ goal was not related to knowledge of goal; $38.2 \%$ of patients aware of their $\mathrm{HbA}_{1 \mathrm{c}}$ goal achieved it vs. $41.4 \%$ of those unaware of their goal ( $p=0.143$ ) (Table 2).

Age, gender, BMI, time since diagnosis, most recent previous $\mathrm{HbA}_{1 \mathrm{c}}$ measure, or Charlson Comorbidity Index [28] did not differ among patients aware and unaware of their $\mathrm{HbA}_{1 \mathrm{c}}$ goal (Table 2). Multivariate logistic regression controlling for these factors showed that patients who were aware of their $\mathrm{HbA}_{1 \mathrm{c}}$ goal were not more likely to meet it than those who were unaware (odds ratio 0.925; 95\% confidence interval 0.721-1.19; $p=0.541$ ).

Patients who were aware of their individual $\mathrm{HbA}_{1 \mathrm{c}}$ goal were using more AHAs in their current treatment regimen than those who were unaware of their $\mathrm{HbA}_{1 \mathrm{c}}$ goal; $68.4 \%$ vs. $55 \%$ of patients, respectively, received more than one AHA $(p<0.0001)$. Those receiving vs. not receiving a GLP-1 receptor agonist, SGLT2i, or insulin were more aware of their $\mathrm{HbA}_{1 \mathrm{c}}$ goal $(p<0.05)$. Patients aware of their $\mathrm{HbA}_{1 \mathrm{c}}$ goal were also on a later line of therapy than patients who were unaware; $53.3 \%$ of patients aware of $\mathrm{HbA}_{1 \mathrm{c}}$ goal were on second-line of therapy or later vs. $44.8 \%$ of patients unaware of goal.

While both patient groups who were aware and unaware of their agreed $\mathrm{HbA}_{1 \mathrm{c}}$ goal reported good adherence to their AHAs, those who were aware of their goal reported slightly higher adherence (ARMS-D total score 14.8 vs. 15.5, respectively; $p<0.0001)$.

\section{Physician-Reported Glucose Testing and Patient Awareness}

Physicians reported that those patients who were aware of their $\mathrm{HbA}_{1 \mathrm{c}}$ goal were more likely to test their blood glucose levels than patients who were unaware $(74.1 \%$ vs. $54.6 \%$; $p<0.0001$ ) (Table 2). Physicians were not satisfied with the current blood glucose level of approximately one third of these patients. They believed that a higher proportion of patients who were aware of their $\mathrm{HbA}_{1 \mathrm{c}}$ goal could achieve better glucose control as opposed to those who were unaware $(32.4 \%$ vs. $28.2 \%$, respectively; $p=0.003$ ). Physicians were more satisfied with the current blood glucose level of patients aware of their $\mathrm{HbA}_{1 \mathrm{c}}$ goal in the subanalysis than those excluded patients not aware of their $\mathrm{HbA}_{1 \mathrm{c}}$ goal $(58.4 \%$ vs. $49.0 \%$; $p<0.0001)$.

\section{Physician Profiles}

The physicians' year of medical qualification and 5-day workload of patients with T2DM did not affect whether their patients were aware of an agreed $\mathrm{HbA}_{1 \mathrm{c}}$ goal or not. However, patients of diabetologists/endocrinologists were more aware of their agreed $\mathrm{HbA}_{1 \mathrm{c}}$ goal than patients of PCPs (75.2\% vs. 67.4\%; $p<0.0001)$ (Table 2).

\section{DISCUSSION}

Major international guidelines recommend the determination of glycemic goals on an individual level based on the respective patient's clinical profile. Data on individual glycemic goals 
and the proportion of patients achieving them are generally not available from large datasets, such as claims data. Therefore, we aimed to assess this important question in a survey of 8794 patients with type 2 diabetes mellitus in the USA and Europe. We found that two thirds of patients were not at the $\mathrm{HbA}_{1 \mathrm{c}}$ goal set by their physician, which was on average $6.8 \%$, and that physicians were not changing/adding AHAs until $\mathrm{HbA}_{1 \mathrm{c}}$ was above $8 \%$. The $\mathrm{HbA}_{1 \mathrm{c}}$ goal of $6.8 \%$ in our analysis is comparable to patient-reported $\mathrm{HbA}_{1 \mathrm{c}}$ goals set by physicians in other countries $(6.1-6.9 \%)$, where $26-70 \%$ of patients reported that they had a specific $\mathrm{HbA}_{1 \mathrm{c}}$ goal [29].

Waiting to change/add therapies could suggest therapeutic inertia, whereby physicians delaying intensification of treatment regimen of patients with type 2 diabetes mellitus when appropriate to achieve good glycemic control [30]. Moreover, it may also indicate that guideline recommendations of a target $\mathrm{HbA}_{1 \mathrm{c}}$ of $<7.0 \%$ [1-3] are not being fully implemented by physicians in clinical practice. Studies have previously found that a considerable proportion of patients with type 2 diabetes mellitus with suboptimal glycemic control experience a delay in receiving treatment intensification with AHAs $[31,32]$. The average time to treatment intensification from one to two AHAs agents in patients with $\mathrm{HbA}_{1 \mathrm{c}} \geq 7.0 \%$ was 2.9 years, 1.9 years in patients with $\mathrm{HbA}_{1 \mathrm{c}}$ $\geq 7.5 \%$, and 1.6 years in patients with $\mathrm{HbA}_{1 \mathrm{c}}$ $\geq 8.0 \%$ [31]. Evidence suggests that patients with type 2 diabetes mellitus do not receive intensified treatment for over a year after monotherapy failure, with half of patients waiting over 5 years [33].

Similarly to our analysis, other studies confirm that patients do not receive treatment intensification until their $\mathrm{HbA}_{1 \mathrm{c}}$ is $>8[33,34]$. Of concern, therapy for around half of patients with an $\mathrm{HbA}_{1 \mathrm{c}}$ of 8 to $\geq 9 \%$ is not intensified [35]. Patients with type 2 diabetes mellitus who intensified treatment earlier have been found to have higher mean $\mathrm{HbA}_{1 \mathrm{c}}$ levels, suggesting that physicians react to disease severity [33, 36]. Early treatment intensification also resulted in patients achieving a greater mean decline in $\mathrm{HbA}_{1 \mathrm{c}}$ level [33, 36]. Moreover, patients receiving rapid treatment intensification appear to achieve a maintained $\mathrm{HbA}_{1 \mathrm{c}}$ reduction faster than patients with delayed treatment intensification or no second-line therapy, despite a higher $\mathrm{HbA}_{1 \mathrm{c}}$ at baseline [37].

Challenges for healthcare systems such as poor communication between healthcare providers, lack of a coordinated care plan, and time limitations may also play a role in inertia with later therapy lines [38, 39].

Over two thirds of patients in our analysis $(68.1 \%)$ were utilizing more than one AHA; most were receiving metformin with fewer on insulin, a GLP-1 receptor agonist, and/or a SGLT2i. A study with a cohort of around 21,000 newly treated patients with type 2 diabetes mellitus (76\% initiated on metformin) in the Republic of Ireland found that about $20 \%$ of those who remained on their initial therapy were non-persistent to their treatment (i.e., treatment gap of more than 12 weeks within 365 days of treatment initiation) [40]. Of those changing treatment regimens, treatment additions were more frequent than changes. After metformin, treatment additions were sulfonylurea followed by a dipeptidyl peptidase 4 inhibitor, and changes were most frequently to a sulfonylurea followed by a metformin combination product [40].

Although the majority of patients $(n=1804$, $70.5 \%$ ) included in the sub-analysis were aware of their $\mathrm{HbA}_{1 \mathrm{c}}$ goal, interestingly, awareness of $\mathrm{HbA}_{1 \mathrm{c}}$ goal did not enhance goal attainment. Other studies have reported that two thirds or more of patients with type 2 diabetes mellitus did not know their last $\mathrm{HbA}_{1 \mathrm{c}}[20,41]$. In one study, the few patients who knew their last $\mathrm{HbA}_{1 \mathrm{c}}$ value reported a biomedically accurate level of diabetes control and better understanding of diabetes care compared with those who did not know their $\mathrm{HbA}_{1 \mathrm{c}}$ value [20]. However, such knowledge of $\mathrm{HbA}_{1 \mathrm{c}}$ did not translate into improved diabetes self-management [20].

With a decline in beta-cell function and mass, more treatments fail to control glycemic levels and there is an increasing risk of the development of complications [42]. Patients may become more aware of their goal if they have more recalcitrant disease because it makes 
goal achievement more difficult. Our analysis indicated that patients who were aware of their $\mathrm{HbA}_{1 \mathrm{c}}$ goal were on more agents and insulin, and to consult with a specialist rather than a PCP. They had also used more lines of therapy and tested their glucose level more frequently. Patients on metformin monotherapy, typically those who are easier to treat, met their goal even though they were less likely to know what that goal was.

Our findings should be considered in light of the survey limitations. The non-random sample of physicians led to over-representation of specialists based on national PCP-to-specialist ratios [43]. Potential differences between PCPs and diabetologists/endocrinologists in knowledge level and diabetes management may have affected patient treatment and clinical outcomes. Furthermore, the patient population was not truly random because of the inclusion of the next ten consecutive consulting patients and two additional patients receiving SGLT2i or GLP-1 therapy. Additionally, analysis of the overall patient population excluded $39.4 \%$ of patients for whom current $\mathrm{HbA}_{1 \mathrm{c}}$ or individualized $\mathrm{HbA}_{1 \mathrm{c}}$ goals were unavailable, or who had been diagnosed with type 2 diabetes mellitus for less than 3 months. Although unsurprising, the reduction of patient numbers evaluated at each change/addition in line of therapy was also recognized. Lastly, this analysis included patients from different European countries and the USA; therefore, findings may have been affected by country variations in clinical practice and may differ should data be drawn from one or more other countries. Further research is required at a country level. Of note, data was collected prior to the emergence of coronavirus disease (COVID-19) and does not reflect shifting practice patterns caused by the pandemic.

In conclusion, we demonstrated that the proportion of patients with type 2 diabetes mellitus achieving their goals for glycemic control remained suboptimal when compared to current guideline criteria. Despite the availability of many new antihyperglycemic medications, about $60 \%$ of patients with type 2 diabetes mellitus did not achieve their individualized $\mathrm{HbA}_{1 \mathrm{c}}$ goal. Intensification of treatment was often delayed until $\mathrm{HbA}_{1 \mathrm{c}}$ was $8 \%$ or higher.
Results of this analysis highlighted the need for a holistic approach to diabetes management, involving patient education, and patient-physician communication and partnership.

\section{ACKNOWLEDGEMENTS}

Funding. Data collection was undertaken by Adelphi Real World as part of an independent survey, entitled the Adelphi Diabetes DSP. Merck \& Co., Inc., Kenilworth, NJ, USA, and Pfizer Ltd., Tadworth, UK, did not influence the original survey through either contribution to the design of questionnaires or data collection. The analysis described here used data from the Adelphi Diabetes DSP. The DSP is a wholly owned Adelphi product. Merck \& Co., Inc., Kenilworth, NJ, USA, and Pfizer Ltd., Tadworth, UK, are two of multiple subscribers to the DSP. The journal's Rapid Service Fee was sponsored by Merck \& Co.

Medical Writing. Medical writing was provided by Sue Libretto, PhD, of Sue Libretto Publications Consultant Ltd (Hertfordshire, UK).

Authorship. All named authors meet the International Committee of Medical Journal Editors (ICMJE) criteria for authorship for this article, take responsibility for the integrity of the work as a whole, and have given their approval for this version to be published.

Author Contributions. DL responsible for clinical oversight and guidance as lead author. Study set-up and data collection were led by VH. Analysis design was conducted by all authors with statistical analyses prepared by GM. DL, RB and $\mathrm{VH}$ wrote the manuscript supported by a medical writer. All authors had access to the aggregated data, participated in manuscript development, provided critical feedback and approved the final manuscript, assume responsibility for the accuracy and completeness of the data, and vouch for the study's fidelity to the protocol. 
Prior Presentation. This is an original work and is not under consideration by any other journal.

The data were presented in part at the Virtual 56th European Association for the Study of Diabetes (EASD) Annual Meeting, September 21-25, 2020: Boggs $R$ et al. Impact of T2DM patients' knowledge of their individualized HbA1c goal on glycemic control; and the International Conference on Pharmacoepidemiology \& Therapeutic Risk Management (ICPE) All Access, September 16-19, 2020: Lautsch D et al. Individualized glycemic control in type 2 diabetes in Europe and the United States.

Disclosures. Dominik Lautsch, Robert Boggs, Tongton Wang, Claudio Gonzalez, Swapnil Rajpathak and Seema Malkani are employees of Merck Sharp \& Dohme Corp., a subsidiary of Merck \& Co., Inc., Kenilworth, NJ, USA, and may own stock and/or hold stock options in Merck \& Co., Inc., Kenilworth, NJ, USA. Euan McLeod is an employee of Pfizer Ltd., Tadworth, UK, and may own stock and/or hold stock options in Pfizer Ltd., Tadworth, UK. Gary Milligan, James Carroll and Victoria Higgins are employees of Adelphi Real World, Bollington, UK.

Compliance with Ethics Guidelines. The survey obtained ethics approval from the Western Institutional Review Board, study protocol number 1247198, and was performed in accordance with the Helsinki Declaration of 1964 and its later amendments. Physicians did not see patient responses, thereby ensuring that future interactions between physicians and their patients were not compromised by patient responses. Patients provided written informed consent for use of their anonymized and aggregated data.

Data Availability. All data relevant to the analysis are included in the article. All data that support the findings of this survey are the intellectual property of Adelphi Real World. The datasets generated during and/or analyzed during the current study are available from the corresponding author on reasonable request.
Open Access. This article is licensed under a Creative Commons Attribution-NonCommercial 4.0 International License, which permits any non-commercial use, sharing, adaptation, distribution and reproduction in any medium or format, as long as you give appropriate credit to the original author(s) and the source, provide a link to the Creative Commons licence, and indicate if changes were made. The images or other third party material in this article are included in the article's Creative Commons licence, unless indicated otherwise in a credit line to the material. If material is not included in the article's Creative Commons licence and your intended use is not permitted by statutory regulation or exceeds the permitted use, you will need to obtain permission directly from the copyright holder. To view a copy of this licence, visit http://creativecommons.org/licenses/by$\mathrm{nc} / 4.0 /$.

\section{REFERENCES}

1. American Diabetes Association. 6. Glycemic targets: standards of medical care in diabetes-2019. Diabetes Care. 2019;42(Suppl 1):S61-70. https://doi.org/10. 2337/dc19-S006.

2. Davies MJ, D'Alessio DA, Fradkin J, et al. Management of hyperglycaemia in type 2 diabetes. A consensus report by the American Diabetes Association (ADA) and the European Association for the Study of Diabetes (EASD). Diabetologia. 2018;61(12): 2461-98. https://doi.org/10.1007/s00125-0184729-5.

3. International Diabetes Federation. Recommendations for managing type 2 diabetes in primary care, 2017. www.idf.org/managing-type2-diabetes. Accessed 01 Mar 2021.

4. Diabetes Control and Complications Trial Research Group, Nathan DM, Genuth S, et al. The effect of intensive treatment of diabetes on the development and progression of long-term complications in insulin-dependent diabetes mellitus. N Engl J Med. 1993;329(14):977-86. https://doi.org/10.1056/ NEJM199309303291401.

5. UK Prospective Diabetes Study (UKPDS) Group. Intensive blood-glucose control with sulphonylureas or insulin compared with conventional treatment and risk of complications in patients 
with type 2 diabetes (UKPDS 33). Lancet. 1998;352(9131):837-53.

6. Holman RR, Paul SK, Bethel MA, Matthews DR, Neil HA. 10-year follow-up of intensive glucose control in type 2 diabetes. N Engl J Med. 2008;359(15): 1577-89. https://doi.org/10.1056/NEJMoa0806470.

7. Cosentino F, ESC Scientific Document Group, et al. 2019 ESC Guidelines on diabetes, pre-diabetes, and cardiovascular diseases developed in collaboration with the EASD. Eur Heart J. 2020;41(2):255-323. https://doi.org/10.1093/eurheartj/ehz486.

8. American Diabetes Association. 9. Glycemic targets: standards of medical care in diabetes. Diabetes Care. 2021;44(Suppl 1):S111-24. https://doi.org/10. 2337/dc21-S009.

9. Dalal MR, Grabner M, Bonine N, Stephenson JJ, DiGenio A, Bieszk N. Are patients on basal insulin attaining glycemic targets? Characteristics and goal achievement of patients with type 2 diabetes mellitus treated with basal insulin and physician-perceived barriers to achieving glycemic targets. Diabetes Res Clin Pract. 2016;121:17-26. https:// doi.org/10.1016/j.diabres.2016.08.004.

10. Yokoyama H, Oishi M, Takamura H, et al. Largescale survey of rates of achieving targets for blood glucose, blood pressure, and lipids and prevalence of complications in type 2 diabetes (JDDM 40). BMJ Open Diabetes Res Care. 2016;4(1): e000294. https://doi.org/10.1136/bmjdrc-2016-000294.

11. Edelman SV, Polonsky WH. Type 2 diabetes in the real world: the elusive nature of glycemic control. Diabetes Care. 2017;40(11):1425-32. https://doi. org/10.2337/dc16-1974.

12. Blonde L, Patel C, Bookhart B, Pfeifer M, Chen YW, $\mathrm{Wu}$ B. A real-world analysis of glycemic control among patients with type 2 diabetes treated with canagliflozin versus dapagliflozin. Curr Med Res Opin. 2018;34(6):1143-52. https://doi.org/10.1080/ 03007995.2018 .1458709$.

13. Blonde L, Brunton SA, Chava PSJ, et al. Achievement of target $\mathrm{A} 1 \mathrm{C}<70 \%(<53 \mathrm{mmol} / \mathrm{mol})$ by US type 2 diabetes patients treated with basal insulin in both randomized controlled trials and clinical practice. Diabetes Spectr. 2019;32(2):93-103. https://doi.org/10.2337/ds17-0082.

14. Robinson S, Boye KS, Mody R, et al. Real-world effectiveness of dulaglutide in patients with type 2 diabetes mellitus: a literature review. Diabetes Ther. 2020;11(7):1437-66. s13300-020-00839-5. https://doi.org/10.1007/

15. Berikai P, Meyer PM, Kazlauskaite R, Savoy B, Kozik $\mathrm{K}$, Fogelfeld L. Gain in patients' knowledge of diabetes management targets is associated with better glycemic control. Diabetes Care. 2007;30(6): 1587-9. https://doi.org/10.2337/dc06-2026.

16. Beard E, Clark M, Hurel S, Cooke D. Do people with diabetes understand their clinical marker of longterm glycemic control (HbA1c levels) and does this predict diabetes self-care behaviours and HbA1c? Patient Educ Couns. 2010;80(2):227-32. https:// doi.org/10.1016/j.pec.2009.11.008.

17. Trivedi H, Gray LJ, Seidu S, et al. Self-knowledge of HbA1c in people with type 2 diabetes mellitus and its association with glycaemic control. Prim Care Diabetes. 2017;11(5):414-20. https://doi.org/10. 1016/j.pcd.2017.03.011.

18. Milo RB, Connelly CD. Predictors of glycemic management among patients with type 2 diabetes. J Clin Nurs. 2019;28(9-10):1737-44. https://doi. org/10.1111/jocn.14779.

19. Gopalan A, Kellom K, McDonough K, Schapira MM. Exploring how patients understand and assess their diabetes control. BMC Endocr Disord. 2018;18(1): 79. https://doi.org/10.1186/s12902-018-0309-4.

20. Heisler M, Piette JD, Spencer M, Kieffer E, Vijan S. The relationship between knowledge of recent HbA1c values and diabetes care understanding and self-management. Diabetes Care. 2005;28(4): 816-22. https://doi.org/10.2337/diacare.28.4.816.

21. Ferguson MO, Long JA, Zhu J. Low health literacy predicts misperceptions of diabetes control in patients with persistently elevated A1C. Diabetes Educ. 2015;41(3):309-19. https://doi.org/10.1177/ 0145721715572446.

22. Finer S, Robb P, Cowan K, Daly A, Shah K, Farmer A. Setting the top 10 research priorities to improve the health of people with type 2 diabetes: a diabetes UK-James Lind Alliance Priority Setting Partnership. Diabet Med. 2018;35(7):862-70. https://doi.org/10. $1111 /$ dme. 13613 .

23. Anderson P, Benford M, Harris N, Karavali M, Piercy J. Real-world physician and patient behaviour across countries: disease-specific programmes-a means to understand. Curr Med Res Opin. 2008;24(11):3063-72. https://doi.org/10.1185/ 03007990802457040 .

24. Babineaux SM, Curtis B, Holbrook T, Milligan G, Piercy J. Evidence for validity of a national physician and patient-reported, cross-sectional survey in China and UK: the Disease Specific Programme. BMJ Open. 2016;6(8): e010352. https://doi.org/10. 1136/bmjopen-2015-010352.

25. Higgins V, Piercy J, Roughley A, et al. Trends in medication use in patients with type 2 diabetes 
mellitus: a long-term view of real-world treatment between 2000 and 2015. Diabetes Metab Syndr Obes. 2016;9:371-80. https://doi.org/10.2147/ DMSO.S120101.

26. Kripalani S, Risser J, Gatti ME, Jacobson TA. Development and evaluation of the Adherence to Refills and Medications Scale (ARMS) among low-literacy patients with chronic disease. Value Health. 2009;12(1):118-23. https://doi.org/10.1111/j.15244733.2008.00400.x.

27. Mayberry LS, Gonzalez JS, Wallston KA, Kripalani S, Osborn CY. The ARMS-D out performs the SDSCA, but both are reliable, valid, and predict glycemic control. Diabetes Res Clin Pract. 2013;102(2): 96-104. https://doi.org/10.1016/j.diabres.2013.09. 010.

28. Charlson ME, Pompei P, Ales KL, MacKenzie CR. A new method of classifying prognostic comorbidity in longitudinal studies: development and validation. J Chronic Dis. 1987;40(5):373-83. https://doi. org/10.1016/0021-9681(87)90171-8.

29. Simacek K, Curran C, Fenici P, Garcia-Sanchez R. Patient perceptions of their glycemic control and its influence on type 2 diabetes outcomes: an international survey of online communities. Patient Prefer Adherence. 2019;13:295-307. https://doi.org/10. 2147/PPA.S186801.

30. Khunti K, Millar-Jones D. Clinical inertia to insulin initiation and intensification in the UK: a focused literature review. Prim Care Diabetes. 2017;11(1): 3-12. https://doi.org/10.1016/j.pcd.2016.09.003.

31. Khunti K, Wolden ML, Thorsted BL, Andersen M, Davies MJ. Clinical inertia in people with type 2 diabetes: a retrospective cohort study of more than 80,000 people. Diabetes Care. 2013;36(11):3411-7. https://doi.org/10.2337/dc13-0331:3411-7.

32. Brown JB, Nichols GA, Perry A. The burden of treatment failure in type 2 diabetes. Diabetes Care. 2004;27(7):1535-40. https://doi.org/10.2337/ diacare.27.7.1535.

33. Desai U, Kirson NY, Kim J, et al. Time to treatment intensification after monotherapy failure and its association with subsequent glycemic control among 93,515 patients with type 2 diabetes. Diabetes Care. 2018;41(10):2096-104. https://doi.org/ $10.2337 / \mathrm{dc} 17-0662$.

34. Fernandes G, Sawhney B, Hannachi H. Distance to glycemic goal at the time of treatment intensification in patients with type 2 diabetes mellitus failing metformin monotherapy in the United States. Curr Med Res Opin. 2020;36(5):741-8. https://doi.org/ 10.1080/03007995.2020.1722623.

35. Pantalone KM, Misra-Hebert AD, Hobbs TM, et al. Clinical inertia in type 2 diabetes management: evidence from a large, real-world data set. Diabetes Care. 2018;41(7):e113-4. https://doi.org/10.2337/ dc18-0116.

36. Paul SK, Klein K, Thorsted BL, Wolden ML, Khunti K. Delay in treatment intensification increases the risks of cardiovascular events in patients with type 2 diabetes. Cardiovasc Diabetol. 2015;14:100. https://doi.org/10.1186/s12933-015-0260-x.

37. Watson L, Das R, Farquhar R, Langerman H, Barnett $\mathrm{AH}$. Consequences of delaying treatment intensification in type 2 diabetes: evidence from a UK database. Curr Med Res Opin. 2016;32(9):1465-75. https://doi.org/10.1185/03007995.2016.1157462.

38. Reach G, Pechtner V, Gentilella R, Corcos A, Ceriello A. Clinical inertia and its impact on treatment intensification in people with type 2 diabetes mellitus. Diabetes Metab. 2017;43(6):501-11. https:// doi.org/10.1016/j.diabet.2017.06.003.

39. Rushforth B, McCrorie C, Glidewell L, Midgley E, Foy R. Barriers to effective management of type 2 diabetes in primary care: qualitative systematic review. Br J Gen Pract. 2016;66(643):e114-27. https://doi.org/10.3399/bjgp16X683509.

40. Grimes RT, Bennett K, Tilson L, Usher C, Smith SM, Henman MC. Initial therapy, persistence and regimen change in a cohort of newly treated type 2 diabetes patients. Br J Clin Pharmacol. 2015;79(6): 1000-9. https://doi.org/10.1111/bcp.12573.

41. Harwell TS, Dettori N, McDowall JM. Do persons with diabetes know their (A1C) number? Diabetes Educ. 2002;28(1):99-105. https://doi.org/10.1177/ 014572170202800111.

42. Fonseca VA. Defining and characterizing the progression of type 2 diabetes. Diabetes Care. 2009;32(Suppl 2):S151-6. https://doi.org/10.2337/ dc09-S301.

43. Hing E, Hsiao C-J. State variability in supply of office-based primary care providers: United States, 2012. National Center for Health Statistics. NCHS Data Brief No. 151, May 2014. https://www.cdc. gov/nchs/data/databriefs/db151.pdf. Accessed 17 Feb 2021. 